\section{$\underset{\substack{\text { hommes } \\ \text { \& migrations }}}{ }$}

\section{Hommes \& migrations}

Revue française de référence sur les dynamiques

migratoires

$1317-1318 \mid 2017$

L'Europe en mouvement

\title{
«Les individus ont une meilleure conscience du monde »
}

Entretien avec Sébastien Thierry, directeur adjoint de l'Agence Erasmus France

\section{Marie Poinsot}

\section{(2) OpenEdition}

12 Journals

\section{Édition électronique}

URL : http://journals.openedition.org/hommesmigrations/3879

DOI : 10.4000/hommesmigrations.3879

ISSN : 2262-3353

Éditeur

Musée national de l'histoire de l'immigration

Édition imprimée

Date de publication : 1 avril 2017

Pagination : 87-91

ISBN : 978-2-919040-38-4

ISSN : 1142-852X

Référence électronique

Marie Poinsot, « « Les individus ont une meilleure conscience du monde » », Hommes \& migrations [En ligne], 1317-1318 | 2017, mis en ligne le 01 avril 2017, consulté le 04 janvier 2020. URL : http:// journals.openedition.org/hommesmigrations/3879; DOI : 10.4000/hommesmigrations.3879 


\section{« LES INDIVIDUS ONT UNE MEILLEURE CONSCIENCE DU MONDE »}

Entretien avec SÉBASTIEN THIERRY, directeur adjoint de l'Agence Erasmus France, réalisé par MARIE POINSOT.

Hommes \& Migrations :

Les programmes Erasmus vont fêter leurs 30 ans cette année. Quels ont été les objectifs assignés à ce programme à son lancement?

\section{Sébastien Thierry :}

Le programme a été adopté par le Conseil des ministres et le Parlement européen en juin 1987. À l'époque, il n'impliquait que onze pays : l'Allemagne, la Belgique, le Danemark, l'Espagne, la France, la Grèce, l'Irlande, l'Italie, les PaysBas, le Portugal et le Royaume Uni. Au cours de la première année de lancement, l'année universitaire 1987-1988, il a permis à 3000 étudiants européens d'effectuer une mobilité à l'étranger. Aujourd'hui, le programme Erasmus est devenu Erasmus + et s'est élargi bien au-delà du public étudiant. En 30 ans, il a bénéficié à plus de 9 millions de personnes en Europe, dont environ la moitié d'étudiants, en permettant à ces personnes d'effectuer un séjour à l'étranger, soit sous la forme d'études ou de plus en plus sous la forme de stage.

\section{H\&M : Au départ, est-ce une volonté des gouvernements ou des universités de développer ce type de programme?}

S. T. : L'initiative a été lancée au niveau de la Communauté économique européenne (CEE), devenue l'Union européenne, pour offrir une possibilité de rapprocher concrètement les peuples. Les universités, qui avaient de tout temps une tradition de mobilité à leur niveau, ont bien entendu embrayé et Erasmus est rapidement monté en puissance.

Dans la mesure où il s'agit d'un programme européen, les fonds sont répartis entre les pays selon des critères qui prennent notamment en compte la population. De fait, les enveloppes budgétaires attribuées à chaque pays sont systématiquement consommées. Les opportunités de mobilités sont en effet extrêmement attractives et il y a beaucoup plus de demandes de bourses que de possibilités d'en financer. C'est bien sûr le cas pour l'enseignement supérieur, aussi bien pour les mobilités d'études que de stage. Mais c'est aussi vrai pour le public des jeunes en formation professionnelle initiale. Ce public, élèves et apprentis de CAP et de bac pro, post-apprentis ou demandeurs d'emploi, a, dès 1995, bénéficié de ces opportunités à travers le programme Leonardo da Vinci, désormais intégré à Erasmus Plus. Pour ces publics, la demande de bourses est extrêmement dynamique et le programme ne peut répondre qu'à une demande sur deux. Bref, c'est aujourd'hui un programme qui rencontre un énorme succès au sein des États participants.

H\&M : Pour la France, quel bilan peut-on tirer du dispositif, en termes de public concerné, de discipline et de pays de destination? 
En termes de destination, le Royaume-Uni est sans surprise le premier pays d'accueil : il concentre près de $30 \%$ des jeunes de la formation professionnelle et un peu plus de $20 \%$ des étudiants. C'est évidemment une donnée importante pour nous dans le contexte actuel du Brexit même si, à l'heure actuelle, nous n'avons aucune précision sur la suite des événements. Mais il faudra à terme sans doute opter pour des destinations alternatives qui, en l'occurrence, sont nombreuses en Europe, l'anglais étant une langue très répandue dans les stages comme dans les études. Les autres destinations sont les grands pays, l'Espagne bien sûr, et l'Allemagne, très demandée pour la formation professionnelle.

\section{H\&M : En dehors des études, quels changements, quelles adaptations doivent faire ces étudiants pour se fondre dans la société dans laquelle ils vont vivre plusieurs mois?}

S T. : C'est un aspect assez invisible mais absolument essentiel dans ce programme. Avant le départ à l'étranger, Erasmus Plus prévoit une évaluation du niveau de langue à travers un outil en ligne (OLS : Online Linguistic Support). Il permet aussi aux étudiants qui en ont besoin d'accéder à des formations en langues. Avoir une mâ̂trise minimum de la langue du pays d'accueil est en effet nécessaire pour une meilleure intégration. Pour les publics de niveau bac et infra bac, une sensibilisation aux aspects culturels a lieu en outre au sein des établissements d'envoi : dois-je dire bonjour en serrant la main, en faisant une bise, deux bises? Comment s'adresser aux gens ou comprendre leurs réactions ? C'est donc une première approche de l'interculturel.

A 80-85\%, les retours de l'ensemble des publics après la mobilité sont très positifs, aussi bien sur les aspects pratiques que de contenu des stages et des études. Ce sont des expériences marquantes et très fortes pour les jeunes en question.

\section{H\&M : Comment se passe le séjour des étudiants étrangers en mobilité en France?}

S. T. : Dans les études que nous avons menées, plus des trois quarts des étudiants estiment les conditions d'accueil en France plutôt bonnes. Il existe cependant des différences d'appréciation selon les régions d'origine: les étudiants allemands et du nord de l'Europe affichent un taux de satisfaction un peu moins bon sur les aspects liés à la pédagogie des enseignements, qu'ils trouvent moins moderne par rapport à ce qu'ils connaissent. À l'inverse, les étudiants en provenance des pays du sud (Espagne, Italie) plébiscitent cette approche, ainsi que les étudiants de l'est de l'Europe, très satisfaits des conditions d'enseignement en France.

\section{H\&M : Savez-vous combien de jeunes ne reviennent pas, ou si certains repartiront dans ces pays qu'ils ont appréciés pendant ce programme?}

S. T. : Une proportion assez faible de jeunes ne revient pas. C'est notamment le cas des stagiaires de la formation professionnelle, généralement de jeunes adultes qui partent sur des durées plutôt longues, de 3 à 6 mois. Ces stages sont souvent pour eux l'occasion de s'insérer professionnellement dans le pays de destination. Ils ne forment cependant qu'une minorité. Car partir avec Erasmus, c'est surtout mieux revenir afin de poursuivre son parcours en France, ou dans le pays de départ en général. Parfois, partir, c'est revenir pour repartir une nouvelle fois. Ainsi, pour les publics prioritaires du programme, pour lesquels la mobilité constitue une difficulté intrinsèque, le fait de partir à l'étranger, même sur des durées courtes (en moyenne 3-4 semaines pour les jeunes de bac pro), constitue un temps de découverte de soi fondamental et de sa capacité à dépasser les obstacles. Le premier de ces dépassements est la langue, mais il y a aussi la capacité à entrer en contact, à s'autonomiser ou à s'adapter à des situations qu'ils n'auraient jamais imagi- 
nées. Ce sont des déclencheurs puissants pour la suite qui permettent à ces jeunes de rendre possible une nouvelle mobilité plus autonome, plus longue, plus tard. La mobilité à l'étranger agit en fait comme un véritable accélérateur personnel, individuel pour ces publics.

\section{H\&M : Les Français sont-ils les plus mobiles en Europe?}

S. T. : Contrairement à une idée reçue, les Français sont très mobiles de façon générale. Si l'on s'en tient au seul programme Erasmus Plus, tous publics confondus, la France est deuxième derrière l'Allemagne-qui dispose dans le programme de fonds plus importants lui permettant de financer davantage de bourses de mobilité. Elle se situe en revanche assez loin devant d'autres pays de taille comparable, notamment le Royaume-Uni. De fait, sur l'enseignement supérieur, nous avons eu la surprise de constater qu'en 2015 que nous avions financé un peu plus d'étudiants que l'Allemagne: quand celle-ci a financé 39719 bourses, la France en a octroyé 39 985. La différence tient dans un mouchoir de poche, mais reste très significative étant donnée la différence de taille démographique entre les deux pays. Donc oui, les Français sont très mobiles.

Autre illustration de cette appétence pour l'international : sur une enveloppe de subvention 2017 de plus de 160 millions d'euros, 125 millions sont fléchés pour le financement de la mobilité. Or, la demande cumulée des candidatures de projets de mobilité reçues début février atteint 218 millions d'euros, soit presque deux fois le budget disponible.

\footnotetext{
H\&M : Vous nous signaliez tout à l'heure que $40 \%$ des mobilités se font grâce à des bourses. Pourquoi, à votre avis les enquêtes qui sont menées mettentelles au contraire en avant un caractère socialement assez sélectif des étudiants dans le cadre d'Erasmus ? Comment faire pour améliorer l'ouverture sociale dans l'accès à ces programmes ?
}

S. T. : Précisément, $39 \%$ des étudiants qui partent avec le programme sont boursiers sur critères sociaux. Mais tous les étudiants qui partent avec le programme bénéficient d'une bourse Erasmus Plus qui se cumule alors avec les bourses sur critères sociaux.

Effectivement, la diversité des publics couverts par le programme est encore insuffisamment perçue, plus particulièrement pour les publics d'élèves et apprentis de CAP et Bac Pro qui sont ceux de l'ex-programme Léonardo da Vinci. Dans l'enseignement supérieur, on ignore aussi qu'un jeune sur cinq en stage Erasmus Plus est un élève de BTS. Or, comme les élèves et apprentis de la formation professionnelle, les jeunes de BTS sont souvent issus d'origines socio-économiques plus modestes que les étudiants des universités et a fortiori des grandes écoles.

Ainsi, parce que cette réalité est mal connue, ces publics pourtant très largement représentés dans le programme passent sous le radar et, trop souvent malheureusement, on entend encore dire que Erasmus Plus est un programme élitiste. Nos chiffres et nos études montrent l'inverse. La dernière en date, "Les notes de l'observatoire $n^{\circ} 3$ : la démocratisation d'Erasmus Plus » décrypte ainsi l'origine géographique, la profession des parents ou encore la situation au regard du handicap des bénéficiaires du programme grâce à un questionnaire auxquels 10000 jeunes de profils très variés ont répondu. Cette étude nous a permis de recueillir un ensemble d'informations particulièrement précises qui nous permet aujourd'hui d'affirmer que le programme travaille pour l'égalité des chances et non pas l'inverse.

\section{H\&M : Ce programme favorise-t-il aussi l'employabilité des personnes diplômées qui y ont participé ?}

S. T. : Oui. Pour s'en rendre compte, nous avons exploité l'enquête Génération du Centre d'études et de recherches sur les qualifications (Cereq) qui mesure les parcours d'insertion des jeunes trois ans après leur sortie de formation. Tous les niveaux 
y sont pris en compte, du décrocheur dans l'enseignement secondaire au thésard qui obtenu son doctorat. L'enquête Génération 2010 concernait tous les sortants de formation cette année-là, soit une population d'environ 700000 jeunes dont 35000 ont été interrogés par le Cereq. L'effet mobilité a pu être mesuré à travers des questions spécifiques et les différenciations de parcours sont flagrantes. Les jeunes sortis de formation dans la population générale mettent 4,3 mois pour trouver un emploi. Pour ceux partis à l'étranger, cette durée moyenne tombe à 2,9 mois. Ils sont plus souvent en CDI pour le premier emploi. D’une façon générale, $70 \%$ des jeunes partis à l'étranger dans le cadre d'une mobilité encadrée (ce qui exclut les séjours de loisir) ont un parcours d'accès durable à l'emploi (CDI plutôt que CDD, temps plein plutôt que temps partiel, etc.) contre $50 \%$ pour ceux qui n'ont pas connu de parcours de mobilité dans leur parcours scolaire ou universitaire. L'effet mobilité sur l'insertion professionnelle est donc aujourd'hui démontré. Nos études s'interrogent désormais sur le pourquoi : que se passe-t-il plus précisément dans le cadre d'un séjour à l'étranger, notamment en termes d'acquisition de compétences mobilisables dans un parcours d'insertion? Contrairement à une idée reçue, ce ne sont pas tellement les compétences métiers ou techniques qui prévalent. Elles font bien sûr partie des acquis d'une mobilité, surtout en stage, et forment un plus dans l'apprentissage d'un métier. Mais nous nous intéressons surtout aux compétences dites transversales, les soft skills. Elles se traduisent par une plus grande ouverture d'esprit, une plus grande adaptation au changement ou à des environnements différents. Elles incluent parfois aussi les compétences dites « citoyennes » : par exemple une meilleure conscience du monde qui nous entoure, des problématiques au niveau européen ou au niveau mondial. La perception qu'ont les jeunes de ces différents acquis montre des évolutions très fortes avant et après la mobilité.

H\&M : Ce programme participe-t-il aussi à l'appropriation d'une identité européenne?
S. T. : À notre niveau, nous n'avons pas de définition de ce qu'est ou est censée être l'identité européenne. Si l'on parle de la sensibilisation à l'Europe et à ce qu'elle fait concrètement notamment à travers un programme comme Erasmus, alors oui, il y a des avancées.

On peut cependant relever que l'on se sent d'autant plus européen qu'on part à l'extérieur de l'Europe et qu'on est finalement perçu par le pays d'accueil non pas comme Slovène, Italien, Polonais ou Français, mais comme Européen. Le programme comprend ainsi un volet international mais celui-ci ne concerne aujourd'hui que l'enseignement supérieur ainsi que le service volontaire européen ; il ne concerne pas les jeunes de la formation professionnelle, ce qui est très dommage. Pour cette raison, l'Agence Erasmus + France plaide auprès de la Commission européenne pour une ouverture du volet international du programme à la formation professionnelle. Nous aurions alors un levier qui permettrait de renforcer ce que l'on peut appeler l'identité européenne, et du moins la conscience d'appartenir à un ensemble politique, institutionnel, culturel à l'échelle du continent.

\section{H\&M : Dans un entretien, Catherine Lalumière, présidente de la Maison de l'Europe de Paris, émet le souhait que la formation à l'Europe soit davantage inscrite en préparation de ces départs. Est-ce qu'il vous semble envisageable que les universités, les centres de formation organisent des modules sur la connaissance de l'histoire de l'Europe, de l'Union et de ses instances?}

S. T. : Cela fait partie ou devrait faire partie des cours donnés aux élèves et aux lycéens. L'Europe est faite d'institutions et s'appuie sur une vie démocratique européenne, notamment avec le Parlement européen, qui est l'expression de la représentation démocratique des citoyens européens, et qui a gagné en pouvoir et donc en capacité d'influence. Mieux comprendre le fonctionnement de l'Union européenne serait souhaitable 
et, dans les préparations avant les départs évoquées plus haut, c'est peut-être un aspect qui gagnerait à être renforcé en complément de ce qui existe dans les cursus scolaires. Ces préparations sont généralement plutôt axées sur des aspects pratiques et pourraient être complétées par un volet «culture politique et citoyenne».

\section{H\&M : Erasmus a-t-il permis de développer les collaborations entre les universités (système de reconnaissance des diplômes, programmes communs de recherche ou d'enseignement) ?}

S. T. : La réponse est à l'évidence oui. C'est même l'un des grands succès du programme Erasmus, surtout au niveau de l'enseignement supérieur. Aujourd'hui, la mobilité en Europe s'est considérablement développée. Elle est devenue " facile ». Un étudiant qui part une année se former à l'étranger dans un autre établissement d'enseignement supérieur voit cette année reconnue dans son parcours grâce au système de crédits ECTS qui permet de valider un semestre ou une année entière. C'est un système qui intéresse d'autres régions du monde telles que l'Océanie ou le Canada pour leur propre programme de mobilité régionale. Ce système est par ailleurs partagé au-delà de l'UE puisqu'il réunit 47 pays.

Cela a été possible grâce au processus de Bologne lancé en 1999. En moins de 10 ans, les Etatsmembres, qui ont été moteurs, ainsi que les établissements d'enseignement ont bâti un système européen basé sur une harmonisation des niveaux de formation, le fameux « 3-5-8 » ou "Licence-Master-Doctorat », ainsi que les crédits ECTS pour la reconnaissance de la mobilité. Cela a permis de renforcer les coopérations entre les universités qui ont aujourd'hui généralement des dizaines - voire des centaines pour certaines - d'accords de partenariat avec d'autres établissements d'enseignement supérieur. Il faut par ailleurs faire en sorte que les échanges s'accentuent, pas seulement au niveau des étudiants, mais aussi au niveau des équipes d'encadrement.
Le programme Erasmus + prévoit ainsi la mobilité des enseignants et des personnels administratifs de façon à ce que l'Europe s'incarne au niveau des systèmes pédagogiques comme administratifs. L'Europe s'est dotée d'une stratégie qui fonctionne pour l'enseignement supérieur et qui permet aujourd'hui qu'une mobilité à l'étranger s'intègre dans un parcours. Mais, sur le champ de la formation professionnelle initiale, du chemin reste à faire. Même si beaucoup de jeunes souhaitent partir à l'étranger faire un stage, la question de la reconnaissance du séjour à l'étranger pour ces publics en est à ses balbutiements. Nous n'avons pas d'harmonisation des systèmes en Europe, les systèmes nationaux restant très peu interconnectés. La forte dynamique de développement de la mobilité pour ces élèves et apprentis de la formation professionnelle, et plus particulièrement le souhait de l'Union européenne de développer des parcours de mobilités longues, devrait permettre progressivement de faire bouger les choses. Une articulation entre les systèmes doit être initiée afin que les jeunes puissent effectuer six mois à un an à l'étranger et voient cette période reconnue dans le cadre de leurs cursus. Ce serait une avancée assez formidable et que l'on peut espérer voir impulsée dans les années qui viennent.

\section{H\&M : Une telle collaboration est importante pour constituer une Europe des universités attractive par rapport aux autres continents, notamment l'Asie qui continue à apprécier la notoriété des formations européennes...}

S. T. : L'Asie, mais aussi l'Afrique. Le système "Bologne " renvoie à la coopération intra-européenne. Erasmus Plus comporte cependant un volet visant plus spécifiquement à renforcer l'attractivité des formations de la région Europe: Erasmus Mundus. Il propose des formations d'excellence élaborées grâce à des fonds du programme. Elles aboutissent à des double ou triple diplômes largement reconnus et très attractifs pour les étudiants du monde entier. 